\title{
Antropología, ecología y minería en las comunidades del área andina
}

\author{
Fabiola YeckTing VILELA \\ Universidad Nacional Mayor de San Marcos (Perú) - \\ Université de la Sorbonne Nouvelle, Paris III (Francia) \\ fabiolayeckting@gmail.com \\ Iván RAMÍREZ ZAPATA \\ Universidad Nacional Mayor de San Marcos (Perú) \\ Ivanandres2008@gmail.com
}

Recibido: 31 de agosto de 2011

Aceptado: 21 de septiembre de 2011

\begin{abstract}
RESUMEN
Este artículo presenta las transformaciones de las comunidades campesinas mineras en los Andes y su problemática en relación con el hábitat y los recursos naturales como producto de los impactos de las industrias extractivas en el área andina. Para ello nos valdremos de herramientas teóricas presentes en discusiones recientes de la antropología ecológica, centrándonos en los cambios en las configuraciones territoriales y comunales, y su dimensión cultural. El artículo tiene por objetivo mostrar casos comparativos del área andina: Tintaya y Cotabambas, en el sur andino del Perú y Oruro y Potosí, en Bolivia, donde los impactos en las poblaciones evidencian la transformación de las condiciones de vida, el cambio cultural derivado de la convivencia entre los proyectos de las industrias y los campesinos, y los impactos en el medio ambiente y la configuración del espacio.
\end{abstract}

Palabras claves: Antropología ecológica, minería, conflicto cultural, comunidades andinas.

\section{Anthropology, Ecology and Mining in the Andean Communities}

\begin{abstract}
This article will present the transformation of rural mining communities in the Andes and its problems in relation to habitat and natural resources as a result of the impacts of extractive industries in the Andean region. For this, we will use theoretical tools displayed in recent discussions of ecological anthropology, focusing on changes in territorial and communal settings, and its cultural dimensions. The article aims to show comparative cases in the Andean region: Tintaya and Cotabambas, in the southern Andes of Peru, and Oruro and Potosí, in Bolivia, where the impact on population is evidenced by the transformation of the living conditions, cultural change of industry coexistence projects and farmers, as well as impacts on the environment and space configuration.
\end{abstract}

Key words: Ecological anthropology, mining, cultural conflict, andean communities.

Sumario: 1. Introducción. 2. Antropología ecológica y debates contemporáneos. 3. Campesinos y mineros en los Andes. Conflicto cultural con relación al medio ambiente y a la dinámica y la tradición histórica. 4. Consideraciones finales. 5. Referencias bibliográficas. 


\section{Introducción}

El tema de la ecología en la antropología, ha sido abordado en diferentes momentos. En una primera etapa, entre las décadas de 1960 y 1970, resaltan las figuras de Steward y White, y se enfatiza el hábitat, el equilibrio de las condiciones de vida, organismos, poblaciones y ecosistemas, y la modificación de su organización, estructura y funcionamiento. Se define el medio ambiente y su entorno proponiendo que la cultura de los grupos produce mecanismos de compensación que permiten los arreglos sociales para su adecuado manejo. Si bien se han registrado ya los quiebres de este devenir de la compleja transformación del ambiente y su relación con la cultura (Towsend 2000), es nuestra intención tomar hitos claves para situar nuestro análisis de los conflictos que genera el reto de la transformación del medioambiente cuando se trata de un territorio de actividad minera en los Andes.

Nos detendremos entonces en la antropología ecológica procesual, como suma y síntesis de debates y corrientes, que muestra la transformación de las identidades a través de la historia y las relaciones ecológicas, donde se contrastan los modelos de vida en relación con la naturaleza y los recursos que utilizan las poblaciones. Se relevan la toma de decisiones y los modelos de las comunidades de base frente a las transformaciones que vienen del exterior. La configuración del espacio/territorio con relación a los recursos naturales y su posterior transformación en las comunidades afectadas por la minería en el área andina, muestran el impacto de la explotación de estos recursos en las condiciones de vida y las relaciones económicas de las poblaciones campesinas rurales.

Se trata de modelos de articulación social y cultural relacionados con la vida comunitaria que se debilitan, transforman o desaparecen paulatinamente, mostrando la necesidad de atención a la problemática de deterioro de los recursos naturales y de las transformaciones en el territorio. Otro momento de la antropología y la ecología da lugar a la reflexión sobre la producción de espacios, donde el ambiente se convierte en «lugar»y se da la conexión entre «lugares», «fronteras» y «mapas» (Hirsch 2004). Se presentan los problemas en la convivencia entre comunidades campesinas y la explotación minera, que además evidencian la fragmentación de los arreglos en torno al espacio, a lo cual se suma la presencia de diversos nuevos agentes y percepciones sobre dicho espacio y sus recursos. En el área andina es posible hacer un seguimiento a los impactos directos en el territorio, el agua y los recursos naturales, siendo los casos más perjudiciales aquellos en los que insumos tóxicos contaminan el entorno como producto del avance de procesos de explotación de los yacimientos, poniendo en riesgo la vida de las poblaciones.

Por ello, nos interesa mostrar las transformaciones de las comunidades mineras en los Andes y su problemática con relación al hábitat y los recursos naturales como producto de los impactos de las industrias extractivas en el área andina. Se trata de una cadena de procesos que: 1) transforman las relaciones de vida comunitaria, 2) modifican el patrón de las relaciones económicas, y 3) reconfiguran el territorio, propiciando el deterioro del medioambiente, afectando a las condiciones de vida y disminuyendo la continuidad de los recursos para las futuras generaciones, lo cual a su vez se inserta en los estudios de ecología política y en las investigaciones sobre 
antropología y minería que plantean el conflicto y diversifican las respuestas de adaptación/resistencia de los actores. Todo ello como producto de la reciente atracción de las inversiones a partir de los años de 1990 y el contexto histórico de explotación de recursos naturales en los países centroandinos.

Desde esta perspectiva, el artículo presenta casos comparativos del área andina: las comunidades de Tintaya y Cotabambas en el sur andino del Perú, y las de Oruro y Potosí en Bolivia. Allí es donde la minería evidencia entre las poblaciones la transformación de las condiciones de vida, el cambio cultural y los impactos en el medio ambiente y en la configuración del territorio. Además, trataremos de mostrar cómo se generan los conflictos en torno a los recursos naturales en la convivencia entre los proyectos de las industrias y las comunidades campesinas.

\section{Antropología ecológica y debates contemporáneos}

Este artículo no busca elaborar un estado de la cuestión sobre la antropología ecológica. Más bien presenta algunas de las principales elaboraciones conceptuales que han surgido en ella a partir de algunos autores distintivos de líneas diferentes de pensamiento, como el neofuncionalismo, la antropología ecológica procesual, y la ecología política, para mostrar cuáles son las razones de los conflictos cuando hablamos de medioambiente y cultura. Así, la elección de estos autores para elaborar esta presentación teórica responde a las diferentes luces que cada uno de ellos dan sobre la relación entre ecología y procesos sociales. Rappaport (1975), desde sus investigaciones en Nueva Guinea, nos ayuda a entender que los cambios en la organización social responden muchas veces a la percepción de una amenaza externa, de forma que el grupo social intenta adaptar su modo de vida a una nueva realidad buscando mantener su orden interno. Desde un recorrido por las diferentes tradiciones, Orlove (1980), en su célebre artículo sobre antropología ecológica, y a partir de sus investigaciones en el altiplano andino, presenta la importancia del cálculo consciente $-\mathrm{y}$ del proceso de decisión entre varias opciones disponibles a elegir- para explicar la interacción entre sociedad y entorno, así como el cambio cultural que puede tener lugar en esta relación, ampliando con ello nuestra visión de la relación entre ecología, ideología y práctica social. De igual manera, Hirsch (2004) muestra que el medio ambiente no es un escenario pasivo sobre el que se desarrolla la conducta, sino que más bien su presencia dentro de las prácticas sociales es explícita, pues la importancia dada por un grupo social a los factores ambientales depende de la relación de aquel con el entorno; ésta relación produce a su vez formas diferenciadas de experimentar el espacio, de acuerdo con los alcances y límites de la acción económica. Por su parte, Descola (2003) relativiza la supuesta universalidad de la oposición Naturaleza/Cultura, y señala la existencia de formas de entender el orden del mundo de acuerdo a otro tipo de criterios cognitivos, lo que implica formas diferenciadas de pensar la relación entre grupo social y medio ambiente. Finalmente, Bebbington (2007) señala que los conflictos socio-ambientales contemporáneos son también conflictos por el territorio, es decir, que la lucha entre comunidades y «agentes de desarrollo» tiene un nudo fundamental en la forma de entender la relación con el territorio, porque es su significado lo que se pone radicalmente en cuestión. 
Estas propuestas no tienen en su centro de interés el desarrollo del conflicto socioambiental, no muestran cómo se desarrolla un conflicto, ni los elementos que pueden llevarlo al éxito o al fracaso. Más bien nos ayudan a pensar situaciones que pueden constituirse en puntos de partida de un conflicto socio-ambiental: cambios radicales en la organización social, que pongan en peligro la integridad del grupo social, la reducción del espacio disponible para la realización de actividades económicas fundamentales, y la disrupción de los significados culturales tradicionalmente asociados a un espacio físico (comunidad), etc.

\subsection{Neofuncionalismo de Roy Rappaport}

Para Rappaport (1975), la ecología estudia las formas de vida de los organismos en relación con su posición dentro de los sistemas mayores que los subsumen. Estudiar los intercambios entre seres vivientes -y entre éstos y su hábitat- implica atender a la conducta; la ecología es, entonces, una ciencia de la conducta y una ciencia biológica. Pero además es una ciencia social, pues las relaciones entre especies diferentes suponen organizaciones diferenciadas de sus respectivos miembros.

Por otra parte, los sistemas vivientes son también cibernéticos: son capaces de compensar o corregir las transformaciones que hagan peligrar alguna dimensión del medio ambiente o de ellos mismos, conservando así el equilibrio del sistema. Algunos sistemas son también adaptativos: modifican su organización, estructura y funcionamiento ante los cambios ocasionados en el medio ambiente. Por eso, para este autor, la cultura es un medio para un fin: elementos usados por poblaciones humanas para satisfacer las necesidades biológicas de los ecosistemas que habitan. De esta manera, la antropología ecológica nos hace preguntar:

«si la conducta emprendida con respecto a las convenciones sociales, económicas, políticas o religiosas contribuye a la supervivencia y bienestar de los actores, o por el contrario los amenaza, y si esta conducta mantiene o degrada los sistemas ecológicos en los que ocurre. Si bien las preguntas se refieren a fenómenos culturales, son respondidas teniendo en cuenta los efectos de la conducta culturalmente informada sobre los sistemas biológicos: organismos, poblaciones y ecosistemas. La característica distintiva de la antropología ecológica no es simplemente que toma en consideración factores ambientales en sus intentos para poner en claro los fenómenos culturales, sino que da significado biológico a los términos clave -adaptación, equilibrio interno, funcionamiento adecuado, supervivencia- de sus formulaciones.» (Rappaport 1975: 267).

Para Rappaport, la preocupación por la evolución no debe de agotarse en los procesos de adaptación o de desarrollo organizativo. Por el contrario, sobre ello prioriza que la evolución puede también generar desadaptaciones, que eventualmente pueden llevar a la muerte del sistema. En este sentido, la flexibilidad cultural puede también hacer las veces de principio desestructurador o disfuncional de un sistema. 


\subsection{La antropología ecológica procesual}

\subsubsection{Benjamin Orlove}

Orlove (1980) explica que el carácter procesual de esta propuesta se encuentra en la importancia que otorga a la historia de las relaciones ecológicas, así como al análisis de los mecanismos de cambio social. Más precisamente, examina las variaciones en actividades grupales e individuales, y cómo las influencias externas y el comportamiento interno interactúan entre sí. De esta forma, se entiende que el medio ambiente no es un telón de fondo pasivo sobre el cual transcurre la acción, sino que tanto ésta como aquél interactúan activamente.

Si bien esta corriente tiene también influencia del neoevolucionismo y del neofuncionalismo, se vale de otras herramientas de análisis en donde lo más importante son los modelos de toma de decisiones de los actores (actor-based models), que permiten ampliar la comprensión de los parámetros de comportamiento de los actores y la racionalidad de sus respuestas ante cambios en las condiciones de vida.

Derivado de los modelos de toma de decisión, la antropología ecológica procesual ha desarrollado diversas herramientas de análisis. Se han producido modelos demográficos de toma de decisiones (demographic decision-making models), examinando así correlaciones entre cambios demográficos y uso y disponibilidad de recursos, o entre cambios demográficos y transformaciones ideológicas. Se ha prestado atención a los problemas ambientales desde el punto de vista de las formas en que poblaciones e individuos responden a ellos, creando tipologías y estableciendo los caracteres particulares de las respuestas de acuerdo con las escalas de los problemas. Se han analizado estrategias adaptativas, y también han sido examinados los patrones de distribución de recursos y los lugares desde donde se fijan las jerarquías de prioridades que regulan tal distribución, así como sus justificaciones ideológicas. Finalmente, los modelos de toma de decisión son excelentes para analizar los mecanismos de cambio social:

«En la antropología ecológica procesual, los modelos de toma de decisiones proveen de un mecanismo de cambio, debido a la interacción existente entre las elecciones que hechas por los actores, los comportamientos a niveles individual y grupal, y los sistemas biológicos, social y cultural que influencian la distribución de recursos, constriñen las posibles estrategias adaptativas, y brindan algunas de las metas que los actores buscan alcanzar. En este enfoque, la cultura y la ideología no son vistas como epifenómenos, sino como causas inmediatas que dan forma a la acción humana. Influencian las opciones disponibles a los individuos para escoger, y, a su vez, son influenciados por las consecuencias acumulativas de tales elecciones.» (Orlove 1980: 257, traducción nuestra).

De esta forma, la perspectiva procesual permite entender mejor las conexiones entre el comportamiento de los actores y los cambios en su medio ambiente, entendiendo las relaciones de cooperación y conflicto, y los cambios en la organización social. 


\subsubsection{Eric Hirsch}

Eric Hirsch (2004) sostiene que hay que superar la idea de que la ecología o el medio ambiente constituyen el telón de fondo pasivo sobre el cual se llevan a cabo las prácticas económicas y, por extensión, la acción social. Por el contrario, economía y medio ambiente constituyen planos que se cortan y se influencian el uno al otro. Así, la acción económica tiene lugar en el marco de un «paisaje moral» (moral landscape), siendo así posible entenderla en términos de escenarios atravesados por lugares, caminos y fronteras de significado que producen formas cognitivas diferenciadas a través los cuales los grupos sociales perciben el entorno y se perciben a sí mismos.

Bajo este enfoque, Hirsch argumenta que si bien factores ambientales como las lluvias o el caudal de un río afectan a la economía, es la relación de las personas con el ambiente lo que traduce e inserta estos factores en la forma económica que una sociedad ha creado. Así, los factores ambientales, a través de su constitución como objetos de conocimiento, se convierten en artefactos mediante los cuales la gente se relaciona entre sí. Los saberes que contextualizan esta relación derivan por lo general de la interpretación de los ciclos estacionales, el ciclo de crecimiento de las plantas, y los relatos y fórmulas mágicas heredadas de los antepasados. En suma, la gente se relaciona y se asigna valor tanto a través de la organización social como a través de la organización del paisaje. Así, un factor importante de las relaciones radica en las maneras en las que «se hace aparecer» objetos, personas y lugares.

Tres entidades son importantes para el estudio de la economía y el medio ambiente: 1) el «lugar» y la conexión entre «lugares»; 2) la «frontera» que establece la distinción dentro/fuera; y 3) el «mapa», que expresa la imagen articulada de «lugares» $\mathrm{y}$ «fronteras». Cada entidad supone una forma específica de socialidad en un espacio local. Los lugares nunca existen en sí mismos, sino en relación con otros lugares por medio de caminos y pasajes. El movimiento entre lugares es lo que les da forma y tamaño, y los convierte en unidades identificables, constituyéndose así las fronteras como entidades que separan lugares y personas unas de otras. La capacidad de las personas para moverse a través de lugares y fronteras supone la existencia -aunque sea únicamente «mental»- de mapas. Así, las tres entidades contribuyen de forma particular a dar forma a paisajes locales. Estas entidades sirven de indicador cualitativo de las formas locales de medida y valor que constituyen un régimen económico (Hirsch 2004: 442).

\subsection{Estructuralismo de Philippe Descola}

Descola (2003) constata que el fundamento clave de la cosmología del pensamiento occidental, la distinción Naturaleza/Cultura, se encuentra en proceso de erosión, por lo que se hace urgente la tarea de desarrollar una antropología renovada: el antropólogo de hoy no debe de estudiar las instituciones culturales en relación con su cercanía o lejanía de la esfera de lo natural, debe más bien concentrarse en los medios que ha empleado la humanidad para objetivarse en el mundo. 
Las combinaciones e intersecciones entre los criterios de interioridad (referente a las imágenes de «alma» y «espíritu») y de materialidad (referido a la forma, las percepciones sensoriales y los procesos fisiológicos) autorizan cuatro ontologías: 1) totemismo, basado sobre la idea de la posesión común de características morales y materiales que definen una esencia identitaria en cuanto tipo singular de un conjunto de grupo humanos y no-humanos; 2) animismo, basado en la idea de que entre grupos humanos y no-humanos existe una interioridad similar pero con diferentes materialidades; 3) analogismo, que afirma la existencia de una similitud de efectos entre «personas» humanas y no-humanas, aunque entre una y otra no haya compartidas ni características «interiores» ni características «exteriores»; y 4) naturalismo, que postula la existencia de una discontinuidad de las interioridades (capacidad para el desarrollo de la cultura) y una continuidad material entre grupos humanos y no-humanos. Esta última ontología es propia del pensamiento moderno occidental, y restringiría la capacidad de elaboración cultural a los sujetos europeos.

Hablar de antropología de la naturaleza supone delimitar como objeto de investigación no sólo a colectivos de seres humanos, sino también al conjunto de seres que interactúan con dicho agregado. El objetivo es estudiar las maneras particulares en que grupos de hombres han conectado y «dado forma» a los sectores de la inmensa red de discontinuidades en los que se localizan («tejer una trama»). Sin embargo, no hay que caer en el error de suponer que «trama» es igual a «cultura». Las tramas son esquemas a través de las que se realiza la construcción social de significado, y como tales deben entenderse como disposiciones psíquicas, sensoriales y emocionales encausadas a la manera de habitus. Permiten así concretar al menos tres tipos de aptitudes: 1) estructurar selectivamente los flujos de percepción recibidos del entorno, asignándoles mayor o menor importancia significativa; 2) organizar las actividades prácticas, emocionales e intelectivas de acuerdo a escenarios más o menos estandarizados; y 3) brindar un marco para interpretaciones típicas de hechos observados. Aquellos esquemas que sean «utilizados» en la mayor cantidad de situaciones y que sobre-determinen el campo de significación según su lógica propia, podrán considerarse dominantes (Descola 2003: 75-77).

\subsection{La ecología politica de Anthony Bebbington}

A razón de que la minería se ha convertido en una actividad de gran importancia política y económica, Bebbington (2007: 26) propone una «ecología política de la transformación territorial en áreas afectadas por la minería» que insertaríamos en la línea de los estudios de antropología política.

El autor argumenta que los lugares y territorios son entidades socialmente producidas: se constituyen en la intersección de los múltiples sentidos que le adjudican actores diversos, y en la conjunción de historias locales y dinámicas globales (capitalistas). Así, los conflictos socio-ambientales son también conflictos por el territorio, definiendo la lucha en un campo amplio de cuestiones tales como: la relación entre ambiente y sociedad que debe de prevalecer en un territorio; quién gobernará el territorio, y cómo; los significados mentados a un territorio; los nexos entre un territorio y 
otro, etc. (Bebbington 2007: 33). Es así que en la producción de territorios participan las estrategias e intereses de múltiples actores (empresas, Estado, movimientos y organizaciones sociales, organismos internacionales, etc.), interactúan dinámicas económicas y políticas, entran en tensión consideraciones sobre desarrollo y medioambiente, y convergen procesos de diversa escala (Bebbington 2007: 35).

Sobre la base de estas consideraciones, Bebbington señala que la expansión de la minería se realiza sobre tierras ocupadas con significados culturales e históricos específicos, y cuya forma de utilización da sustento a sus pobladores. En este sentido, estaríamos ante dos enfoques opuestos del territorio: uno que implica una gobernanza territorial (el proceso a lo largo del cual se definen y aplican las reglas que determinan el acceso, control y uso de los recursos naturales) con presencia de diversos actores en él, y otro que garantice la ocupación de un único actor. Este segundo modelo supone, por dos razones, un cambio drástico y arriesgado en la vida de las poblaciones involucradas en él. Por un lado, modifica de manera inmediata el tipo de intervención a realizar sobre el territorio, así como la distribución del poder dentro del espacio local, lo cual afecta tanto a la organización social local como a la disponibilidad de recursos y al medio ambiente. Por otra parte, no sólo es una lógica distinta, sino que este nuevo sujeto de poder es externo a la comunidad -y seguramente ni conoce ni se interesa en conocer las formas de vida y las necesidades locales-, por lo que impone una dinámica propia y, la mayor parte de las veces, nociva para la población (Bebbington 2007: 24-25).

A continuación, evaluaremos todas estas propuestas teóricas en las investigaciones sobre minería en los países andinos.

\section{Campesinos y mineros en los Andes. Conflicto cultural con relación al medio ambiente y a la dinámica y la tradición histórica}

La transformación de las condiciones de vida en las comunidades andinas mineras replantea no sólo la relación hombre-naturaleza en las principales zonas actuales de expansión de la industria, sino también los arreglos sociales, económicos, políticos y culturales de las poblaciones, resignificando las estrategias de subsistencia y la disputa por los territorios y su configuración. En el Perú es significativa la reciente expansión minera en todo el país a partir de los años 1990, pero es de particular interés, por la interrelación entre las identidades y la relación con el medio ambiente, en los departamentos del sur andino como Cuzco o Apurímac. En otros países andinos como Bolivia, de larga tradición histórica minera, la minería convive con la vida comunitaria y la organización en ayllus, aún con los problemas que ello acarrea; a su vez, muestra las transformaciones que han sufrido la organización comunal y las relaciones comunales que demandan nuevos arreglos frente al deterioro de los recursos, especialmente al avance de la contaminación del medioambiente y de las fuentes de agua. 


\subsection{Minería y transformación de las comunidades campesinas en Tintaya}

En el departamento de Cuzco, en la provincia de Espinar, los antiguos pobladores de la comunidad campesina Antaycama y de su anexo Tintaya Marquiri ya tenían conocimiento de la existencia de cobre en la zona. De hecho, el nombre de la comunidad madre de Antaycama deriva de los vocablos quechuas «anta», cobre, y «cama», abundante. En su origen precolombino, la comunidad campesina de Tintaya Marquiri estuvo poblada por diversos grupos étnicos, constituyendo parte del territorio de la nación k’ana con influencia aymara (CooperAcción 2001: 14).

La población de la comunidad, debido a la reciente etapa de violencia subversiva que sufrió el país y a sus propias experiencias de negociación con las empresas mineras, se ha reducido significativamente. De hecho, la comunidad campesina de Tintaya-Marquiri fue creada en 1980, como consecuencia del desmembramiento de la comunidad de Antaycama, lo que dio lugar a la conformación de diecisiete nuevas comunidades (Decouster et al. 2004: 15). Para 2001 eran 180 comuneros empadronados y 60 no empadronados, con una población total aproximada de apenas 1.000 personas. La empresa minera BHP Tintaya, que operó en la mina de Tintaya y que se vendió a la empresa Xtrata en el año 2004, se encuentra a poca distancia de esta comunidad de Tintaya-Marquiri, y explota uno de los yacimientos de cobre más importantes en el Perú.

Las condiciones de vida se transformaron profundamente a partir de la explotación de los recursos mineros en la zona de Tintaya. Cambiaron la propiedad de los recursos y con ello los patrones de vida campesina. El gobierno peruano expropió 2.368 hectáreas de tierras a Tintaya-Marquiri, uno de los principales anexos, estimándose que 86 familias quedaron sin tierras (Aroca 2008: 138-139). El desarrollo de la actividad minera en Espinar, provincia del departamento de Cuzco, implicó en algunos casos que comunidades campesinas perdieran sus tierras por expropiación directa de parte del Estado peruano; también por procesos de venta donde los miembros de las comunidades actuaron bajo presión.

Entre los años 1990 en los que se inició la operación minera y la posterior resistencia de las diversas organizaciones, se dio por ejemplo la formación del Consejo Regional de Comunidades Afectadas por la Minería CORECAMI Cuzco, así como la movilización y protesta de diferentes sectores de la población en Espinar. En esos años también surgió el sentido histórico de identidad, con líderes agrupados en un partido político llamado Minka (Movimiento de Integración K'ana), movimiento que buscaba restablecer la identidad original de los k'ana, la cultura pre-inca en esa zona (Muñoz et al. 2006: 16). Se transformó la gestión política, fortaleciendo la organización comunal y los cargos rotativos, lo que sirvió, junto con otros procesos, para la toma de decisiones sobre el manejo de los recursos y del espacio. El liderazgo desde dentro impulsó un modelo propio de negociación de la vida en las comunidades, reafirmando la identidad cultural como clave en la articulación de una propuesta campesina con base en la identidad cultural. También se logró la intervención de diversos agentes, entre ellos el Defensor del Pueblo, y de la ONG internacional Oxfam con sede en Australia, quienes realizaron diversos diagnósticos e intervenciones. Así, se llegó a la conclusión de que: 
«Los conflictos que se desarrollaron en Espinar en esos años fueron motivados por las demandas de las poblaciones afectadas por las denominadas «ventas forzadas». La pérdida de tierras comunales a favor de la minería no previó las condiciones necesarias para la subsistencia posterior de los comuneros [cfr. CooperAcción 2001], se valorizó la tierra con montos ínfimos, se incumplieron compromisos, hubo informalidad en las concesiones, se realizó tratos con directivos no autorizados; se ejerció presión con solicitudes de servidumbre minera en trámite; se produjeron desalojos forzados, abusos contra las mujeres, entre otros problemas» (Mining Onmbusman: Annual Repport 2001-2002, Community Aid Abroad, November 2002, cit. in Cuadros 2010: 39).

En las comunidades de Tintaya, como producto de la venta de tierras, se dio una transformación en la vida de los miembros de las comunidades afectadas; se quebraron la continuidad de la propiedad de la tierra de las comunidades y las condiciones de subsistencia. La adaptación al cambio -como diría Rappaport- se convierte en una dinámica que afecta a la continuidad del hábitat y de sus recursos. Una de las principales características de los nuevos conflictos es la disputa por los recursos naturales en las nuevas comunidades mineras en el área andina, donde antes de los proyectos se reproducían patrones de vida agropecuaria, también como producto histórico de la dinámica de las comunidades en las haciendas y después de la Reforma Agraria, con relación a los recursos naturales que estaban a disposición de las poblaciones campesinas en sus espacios rurales. La desadaptación significa replantear modelos de comunidades y de relaciones económicas sobre la base de los recursos que son ahora utilizados por las empresas. Los beneficios que los proyectos reciben por el uso directo de estos recursos ya no son percibidos por las poblaciones de las comunidades. La construcción de modelos de comunidades con características de toma de decisiones planteados por los neoevolucionistas y la antropología ecológica procesual sobre la base de la situación de sus recursos se quebrará frente a la irrupción e intervención de los otros actores.

Así, en setiembre del año 2003, después de varias mesas de diálogo entre distintos actores de la región y la empresa BHP Billiton, y de protestas en Espinar, se decidió realizar un Convenio Marco que se convirtió en un referente de responsabilidad social de la empresa y un logro de las organizaciones que participaron, y que es hasta la actualidad un referente tanto en la región como internacionalmente. Sin embargo se producen constantemente nuevos desacuerdos con las acciones del proyecto de explotación con relación a aspectos como el cuidado del medio ambiente.

Actualmente, el proyecto - que ya va por su cuarta etapa- presenta en la zona problemas de contaminación del agua a causa de los relaves, falta de producción agrícola, escasez de agua, desaparición de peces en los ríos y con ello aparición de diferentes enfermedades en personas y animales (Cuadros 2010: 57) ${ }^{1}$. Además se proyecta construir un mineroducto que se articule con el proyecto de la zona de las Bambas, que tendría una planta de relaves en Tintaya que la población rechaza. Se puede comparar entonces cómo el rediseño de estrategias frente al cambio de la vida cotidiana, y los retos que representa en las poblaciones afectadas recientemente por la minería en los espacios rurales con proyectos que tienen un período de explotación de más de diez años, muestra el impacto de los agentes externos e implica arreglos en el medioam-

1 Esta autora pone especialmente en evidencia los problemas de las mujeres por la presencia de la mina, estableciendo una comparación entre Tintaya y Las Bambas. 
biente, así como adaptaciones que han supuesto procesos de cooperación y de resistencia a las transformaciones del territorio y el deterioro de sus recursos.

\subsection{Comunidades campesinas y mineros en Grau y Cotabambas}

Existe una suerte de continuidad de runakunas de identidad quechua en la zona que corresponde a las provincias de Grau y Cotabambas, en el departamento de Apurímac, colindante con la región de Cuzco. Muy cerca de su área de influencia se desarrolló a principios del siglo XVI la minería aurífera en Progreso, en un distrito denominado Qochasayhuas, que de manera progresiva tuvo un fuerte impacto en el medioambiente, en la vida social, económica y cultural, lo cual, con la creación del distrito de Progreso, trajo consigo una idea de modernidad y de desarrollo temprano en la región.

Las historias de vida de los protagonistas con las experiencias de relación directa con la mina y la vida campesina, muestran sus percepciones respecto de las entrañas de los cerros, antes considerados como seres protectores en las minas de Qochasayhuas (Valderrama y Escalante 1992: 214). En la década de 1950 se registraron rituales que, según testimonian los actuales pobladores del distrito de Challhuahuacho, todavía practicaron junto a sus padres y abuelos, como por ejemplo rituales para la siembra y la cosecha, o para alejar fenómenos naturales como la granizada.

Desde el año 2004, el proyecto Las Bambas se ubica en el departamento de Apurímac, entre las provincias de Grau (27.574 pobladores aprox.) y Cotabambas (44.028 pobladores aprox.). Entre los distritos de Progreso, Couyllurqui, Tambobamba, Huyallati y Challhuahuacho, que forman parte del «Cooper Belt del Sur» ${ }^{2}$, existen otros proyectos como Tintaya, Quechuas y Antapaccay. El conjunto lo integran los yacimientos de Chalcobamba, Ferrobamba, Sulfobamba y Charcas, con un área total de concesión minera de aproximadamente 33.200 hectáreas (Cuadros et al. 2005: 11).

Del conjunto de tradiciones que caracterizan la interrelación comunal en Cotabambas, se mantienen las fiestas, carnavales, pagos a la tierra, tinkas, música, danza, tejidos iconográficos, comida, medicina tradicional y relaciones de parentesco que son la base de la estructura de asentamiento poblacional. Una de las mayores riquezas culturales de la zona son sus tradiciones orales y, en la zona de las Bambas, el conocimiento ancestral de variedades de cultivos, en especial de la producción de papa ${ }^{3}$. Sin embargo, paulatinamente se está abandonando la tradición agrícola de la zona.

En el marco del proyecto Las Bambas, de Xtrata, se estudia la tradición ritual de una de estas comunidades, Fuerabamba, próxima a ser desplazada y reubicada. Se trata fundamentalmente de rituales de agradecimiento a la naturaleza y sus recursos, como t'inkas y t'ikachay («reatillas» de colores en las orejas de los ganados), ocultamiento en los cerros de piedras sacadas de los ríos que se envolvían en un tejido blanco (unkuña) para asegurar la producción, pagos a la Pachamama, sacrificio de

\footnotetext{
2 Cinturón de Cobre del Sur, espacio interconectado de presencia del mineral en el sur andino. Comprende otros importantes proyectos de explotación, como La Escondida y Chuquicamata en Chile.

3 En su trabajo de los años 1970, Valderrama y Escalante (1992) recogen que cada campesino siembra alrededor de 80 variedades de papas en sus chacras, las cuales clasifican.
} 
animales, y ofrendas a los cerros, entre otros (Glave et al. 2007: 101).

Los actuales miembros de las organizaciones y autoridades destacan la vigencia de las costumbres de la vida en la comunidad como el ayni, los carnavales, la t'inka, la siembra de la chacra y la ganadería ${ }^{4}$. Ello se articula con otras tradiciones culturales como la vestimenta o el uso del quechua, que reaparecen en el discurso de identidad local y especialmente en el de los líderes tradicionales de origen campesino. Sin embargo, la población percibe a su vez que las transformaciones en torno al medio ambiente y a sus recursos han modificado sus formas de vida desde la introducción del proyecto minero. De esta manera, se pregunta por la eficiencia de los sistemas de producción agrícola frente a la explotación minera. Los campesinos de la zona que mantuvieron actividad agropecuaria muestran su asombro por la falta de atención al problema del riego y de las fuentes de agua. Así una de sus autoridades pone en evidencia en el año 2009 el conflicto que surge en la relación con los recursos, antes de la aprobación de la etapa de explotación del proyecto:

«Todo está llevando a un fin a favor de la minería, aquí en el distrito de Cotabambas, Choquecca, Pamamarca y el río. La minería en el distrito para mi sería negativa, todo sería para la gran empresa, el discurso de una tecnología moderna solamente es un engaño para mi, todas las consultas cuando aprobamos, lo hacemos con policías, con armas como sea...Ahora cuando convoca es similar, quién reina es Xstrata, están sembrando cemento. Los de Fuerabamba, son doce o catorce comunidades, porque en el proyecto está 36.000 Has son cabecera de cuenca entonces cuánto va a afectar eso hacia Tambobamba, Chalhuahuacho, Coyllurqui». (Entrevista a Víctor Limaypuna, teniente de alcalde y regidor de la municipalidad provincial de Cotabambas; septiembre de 2009).

Una de las mayores preocupaciones de la población campesina es el mantenimiento de las fuentes de agua. Aún en la actualidad existe incertidumbre respecto de las transformaciones que se dan en el espacio y en la relación de la vida comunitaria, cuyo eje actual es esa comunidad de Fuerambamba en proceso de desplazamiento. De esta manera se acelera el cambio y se sientan las bases de una transformación del espacio agrícola en espacio minero.

En este caso de reciente etapa de explotación del proyecto ya no sólo se trata de los recursos naturales y de cómo se ha dado la transformación del espacio con relación al desplazamiento de la población, sino de la transformación de la articulación que Hirsch señala de la economía natural y de las relaciones económicas dentro del espacio; si bien hasta la etapa de las haciendas eran de base agropecuaria, en la actualidad se han convertido en mineras. Se declaran, de este modo, los distritos mineros y se produce la desconexión entre el lugar, la frontera y el mapa.

Fuerabamba, esa comunidad que será desplazada del área de explotación, ya no existirá más, y el mapa del distrito se alterará de tal manera que además su población entrará en competencia por los beneficios de la empresa. Es aquí cuando se ponen a prueba la articulación de las ideologías, relacionadas con la identidad, y los meca-

\footnotetext{
4 Testimonios recogidos en el Taller sobre el Manejo de los Recursos Productivos en colaboración con CooperAcción y el Centro Bartolomé de las Casas, a partir de trabajos de campo realizados en noviembre de 2008, en Chalhuahuacho (Cotabambas, Apurímac).
} 
nismos de conflicto/cooperación a los que se refiere la antropología procesual y que bien resume Orlove. En las diferentes estrategias de adaptación que se diseñan, la población local se adapta y a su vez resiste a la nueva distribución y el nuevo gobierno de unos recursos que ya no son comunales, y en los cuales las opciones de toma de decisiones son limitadas por la nueva configuración del espacio y de la administración de los recursos. Habrá que renegociar las nuevas condiciones de modernidad y progreso que están ligadas a la dinámica minera con un rediseño del distrito a partir del tajo que se instalará en el espacio de la comunidad desplazada, y que se reubicará en otro espacio denominado como Nuevo Fuerabamba.

\subsection{Minería y medio ambiente en las comunidades de Oruro y Potosí en Bolivia}

Una de las principales referencias sobre minería en los Andes es el Cerro Rico de Potosí, cuyas reservas de plata se empiezan a explotar en la colonia. Desde la temprana explotación del también llamado «Cerro Rojo» se evidenciaron los procesos de subordinación, conflicto y resistencia entre mineros y campesinos indígenas. En la etapa colonial fueron la plata y el mercurio los minerales que más se explotaron en la región andina (Contreras 2003), impulsando la transformación de la población indígena en los denominados indios de faltriquera (Bakewell 1984: 161) ${ }^{5}$, aquellos que eran contratados en reemplazo de los mitayos, miembros de las comunidades indígenas obligados a trabajar en la mina. En Potosí se generó un circuito económico y comercial que vinculó todo el Virreinato del Perú, actuando el centro minero como polo de atracción en la ruta Buenos Aires-Alto Perú.

Según Absi (2005), la identificación del Cerro Rico con la Pachamama es notoriamente específica de Potosí. Los campesinos de Bolivia y Perú que invocan al Cerro Rico en sus rezos se dirigen a la montaña y no a la Pachamama. De manera más general, ésta última es considerada como la esposa de los espíritus masculinos de las montañas (Harris y Bouysse-Casagne 1987: 263). Los rituales y las ch'allas subterráneas están principalmente destinados a la Pachamama y al Tío, otro ser mítico representado como el diablo, y que habita en las entrañas del socavón. Aquí encontramos esa suerte de continuidad de la naturaleza ontológica a la que se refiere Descola (2003), y que se aplica en el área andina a la relación con los seres naturales que, incluyendo al hombre, forman parte del espacio y de la experiencia de la relación con la naturaleza. Las características que hemos señalado son las del espacio donde se abre la mina en el socavón, generalmente en el lugar de las grandes montañas de donde se extrae el metal.

En el norte de Potosí se sostuvo una suerte de continuidad en las relaciones entre los ayllus como complejas unidades de organización territorial y de parentesco, donde -según se ha sostenido-ni las reformas liberales del siglo XIX, ni el efecto modernizador del sindicalismo y la Reforma Agraria (Rivera 1990: 12), ni la influencia del impulso de los proyectos de desarrollo de los años 1990, han logrado desestructurar. Con los sindicatos existió una articulación entre la creencia religiosa y el compor-

5 Destacan en este trabajo de Bakewell las comparaciones entre las minerías de los Andes y de México. 
tamiento político; se transforman los significados de pertenencia como las nuevas ideologías, los ritos y la conciencia de clase (Nash 1985: 128).

Sin embargo, se ha continuado una tradición de explotación de los minerales que trajo consigo un largo proceso de deterioro del medio ambiente y los recursos naturales. Así, los testimonios entre la población de los ayllus de Potosí evidencian una preocupación de la población por la contaminación de sus recursos:

«Antes la contaminación no había. Cuando han entrado las empresas transnacionales, ellos han traído contaminación modernizando el trabajo con maquinarias porque antes las cooperativas y las empresas no sabían trabajar con tecnologías, las tecnologías traen la contaminación ambiental... Por eso siempre decimos (tenemos que cuidar al medio ambiente) como sabían cuidar nuestros abuelos, los usos y costumbres. Todos somos contaminadores, si compramos bolsas de nylon, eso se va sumando, eso es la contaminación, por eso es que algunos indígenas no utilizamos nylon ni nada. Todos hay que investigar para minimizar el daño a nuestro medio ambiente». (Entrevista a Leandro Tapia Choque, promotor ambiental del ayllu Machaca. «Contaminación minera: Potosí escucha alternativas». PIEB Periódico Digital de Investigación sobre Bolivia. http://www.pieb.com.bo/03-05-2010).

En las comunidades de Oruro y de Potosí, en la cuenca del río Pilcomayo, la contaminación generada por los ingenios o plantas metalúrgicas, deviene principalmente de la descarga de colas con contenidos de minerales sulfurosos y del uso de reactivos químicos, productos orgánicos y otros, en operaciones de concentración, cuyos efectos negativos repercuten directa e indirectamente en el hombre y en la biodiversidad. (Gutiérrez Agramont 2009: 6). El actual deterioro responde además a la continuidad de la explotación de los recursos minerales que presenta impactos en las principales fuentes de agua y en los recursos naturales. En Bolivia se considera que alrededor de 120 municipios de Potosí, Chuquisaca y Tarija están afectados por la contaminación de la rivera del Pilcomayo (Gutiérrez Agramont 2009:14).

En Oruro, después de los sindicatos y el proceso de Reforma Agraria, los ayllus han continuado en un proceso de cambio. Sin embargo, en Oruro se habla del conocimiento de las tres pachas: alaxpacha (universo), manaqxapacha (dentro de la tierra, subsuelo) y aqapacha (superficie de la tierra), que también se conoce como «territorio». Esta última es la base fundamental de todos los seres: el territorio da la vida con su alimentación diaria. Así, su organización guarda relación con el sistema de repartición de terrenos en zonas agrícolas húmedas o secas, denominadas mantas, supervisada por las autoridades (Choqueticlla et al. 2001:63). A través de la organización de la Federación de Ayllus del Sur de Oruro (FAVOR), que forma parte del Consejo Nacional de los Ayllus y Markas del Qullasuyu (CONAMAQ), se reivindica la defensa de los derechos a la identidad cultural, el reconocimiento de tierra y territorio indígenas que comprende recursos naturales y hábitat, y el derecho a la autogestión y a las formas de desarrollo que comprendan la forma de vida y de organización basada en el ayllu.

Sin embargo, en Bolivia entera se registra el deterioro de los recursos naturales. En Oruro y Potosí la minería es la principal actividad, producto de una tradición económica que data de la explotación minera en la Colonia que luego se extiende a la minería del estaño en la época republicana y, actualmente, a la explotación de minerales complejos como plomo, plata, zinc y también oro, como eslabones importantes de un 
proceso de estructuración del espacio regional. Producto de esta especialización regional, se han invisibilizado otros aspectos del medio natural, como su biodiversidad y sus potenciales agropecuarios, propios de los ecosistemas de altura que lo caracterizan (Coronado 2008, cit. in Gutiérrez Agramont 2009). Es así que también se pone en cuestión la forma de gobernanza y la toma de decisiones sobre el territorio que la población y las autoridades deben articular para enfrentar los daños ya producidos y que atribuyen a la mayor modernización y tecnificación después de las cooperativas.

\section{Consideraciones finales}

Las diferentes vertientes de la antropología ecológica sirven para analizar los impactos de la minería en la relación con el hábitat, la disposición de los recursos y la organización de las poblaciones en el territorio. También sirven para analizar el replanteamiento de las relaciones económicas, los conflictos culturales y la disputa por el gobierno de los territorios, lo que implica la intervención de agentes y presiones externas en la toma de decisión y la disrupción de los modelos basados en la vida en las comunidades.

Como hemos visto, la recogida de información evidencia que el origen de los conflictos está en la transformación del medioambiente como espacio de interrelaciones biológicas y en la disputa por el territorio, como el locus de articulaciones económicas, políticas y culturales. Así, se presentan diversas causas y escenarios. Se dan los casos de Tintaya y Cotabambas, con el quiebre de las relaciones económicas y de espacios comunales que ya no lo son más y que obligan a rediseñar el territorio y competir por los recursos naturales escasos y deteriorados, como las fuentes de agua. A su vez, se plantean desafíos al reubicar comunidades y con ello desarticular sus tradicionales formas de vida y el diseño del espacio. Evidenciar dicha transformación nos hace reflexionar en el tránsito planteado desde las estrategias de adaptación a las políticas de gobernanza del territorio; un campo poco explorado el de las experiencias ontológicas y sensoriales con la naturaleza que configuran el mundo de los rituales y de los entramados que subjetivan dicha relación. Sin embargo, por las expectativas que genera la modernidad -que implican el progreso económico de la extracción de los recursos-, las poblaciones se ven en la necesidad de cuestionar directamente el impacto de las industrias, así como de transformar las prácticas culturales con relación al espacio y sus recursos en las zonas de impacto de los proyectos. Así, en los espacios donde la tradición minera ha coexistido con la organización comunal a través de los ayllus -como en el sur de Oruro y el norte Potosí- se vuelven los ojos a las tradiciones y a las economías locales basadas en los recursos naturales, para analizar en qué medida el deterioro y la contaminación que producen los metales en la cuenca del río Pilcomayo se contraponen con las dinámicas productivas locales.

En medio de los procesos de conflicto/negociación emerge la disputa por la continuidad del territorio y el manejo de los recursos en las comunidades andinas. Se replantea así la relación con los espacios ecológicos politizados a partir de a) toma de decisiones, b) tipos de organización de defensa de antiguos modelos de articulación económica y c) necesidad de participación en el gobierno y el diseño de políticas so- 
bre los recursos y el territorio. Quedaría entonces pendiente investigar en qué medida las comunidades logran rediseñar sus estrategias e interrelaciones con el medioambiente y el territorio sin que ello signifique el surgimiento de nuevos conflictos.

\section{Referencias bibliográficas}

Absi, Pascale

2005 Los ministros del diablo: el trabajo y sus representaciones en las minas de Potosí. La Paz: Institut Francais d'Études Andines (IFEA) - Institute de Recherche pour le Développement (IRD) - Embajada de Francia en Bolivia - Fundación PIEB.

Aroca, Javier

2008 «El caso de Tintaya», en Defendiendo derechos y promoviendo cambios. El estado, las empresas extractivas y las comunidades locales en el Perú, Martin Scurrah, ed., pp. 137-197. Lima: Instituto de Estudios Peruanos - OXFAM América - IBC.

BAKEWELL, Peter

1984 Miners of the Red Mountain. Indian Labor in Potosi, 1545-1650. Albuquerque: University of New Mexico Press.

BeBBington, Anthony

2007 «Elementos para una ecología política de los movimientos sociales y el desarrollo territorial en zonas mineras», en Minería, movimientos sociales y respuestas campesinas. Una ecología política de transformaciones territoriales, Anthony Bebbington, ed., pp. 23-46. Lima: Instituto de Estudios Peruanos - CEPES.

Choqueticlla, Vicente, Juan Maraza y Nimeón Vásquez

2001 «Del fortalecimiento del ayllu a la reconstrucción de la nación Quillacas-Azanaques», en Doce experiencias de desarrollo indígena en América Latina, T. Carrasco, D. Iturralde y J. Uquillas, coords., pp. 59-75. Quito: Banco Mundial.

CooperAcción

2001 Los conflictos de tierras en la provincia de Espinar: el caso BHP y las comunidades de Tintaya, Marquiri y Alto Huancané. Lima: CooperAcción.

Contreras, Carlos

2003 «Vida y trabajo en las minas del Perú de la época colonial», en Territorio, cultura e historia. Materiales para la renovación de la enseñanza sobre la sociedad peruana, Patricia Oliart, ed., pp. 95-107. Lima: Instituto de Estudios Peruanos.

Coronado, Felipe

2008 Conflictos socio-ambientales en la gestión de los recursos hídricos y suelos. Documento de comentarios. Convocatoria contaminación minera departamento de Oruro - PIEB. La Paz: PIEB.

CuAdros, Julia

2010 Impactos de la minería en la vida de hombres y mujeres en el sur andino. Los casos de las Bambas y Tintaya. Una mirada desde el género y la interculturalidad. Lima: CooperAcción. 
Cuadros, Julia, Jaime Consiglieri y José de Echave

2005 Mapeo de la situación social e institucional del proyecto Las Bambas. Lima: CooperAcción.

Decouster, Jean-Jacques, Eliana Lia Rivera Alarcón y Karina Baca Gómez Sánchez

2004 Gobernanza ambiental y territorial en comunidades afectadas por la explotación minera. La experiencia de las comunidades campesinas de Espinar-Perú. Cuzco: Asociación Kuraka.

Descola, Philippe

2003 Antropología de la naturaleza. Lima: Instituto Francés de Estudios Andinos - Lluvia Editores.

Glave, Manuel, Gerardo Damonte, Margarita Huamán, Cristina Rosemberg y Luis Escobedo 2007 Línea de base social y económica de las comunidades del área de influencia directa de Xstrata S.A. en el ámbito de la operación minera Las Bambas. Informe final elaborado por el Grupo de Análisis para el Desarrollo. Lima: Grade.

Gutiérrez Agramont, Rita

2009 Contaminación minera en Oruro y Potosí. Estados de Investigación Temática. PIEB. Programa de Investigación Estratégica/ Programa de Investigación Ambiental. La Paz: Fundación PIEB.

Harris, Olivia y Terréese Bouysse-Cassagne

1987 «Pacha: en torno al pensamiento aymara», en Tres reflexiones sobre el pensamiento andino, T. Bouysse-Cassagne, O. Harris, T. Platt y V. Cereceda, eds., pp. 11-59. La Paz: Hisbol.

HIRSCH, Eric

2004 «Environment and Economy: Mutual Connections and Diverse Perspectives». Anthropological Theory 4 (4): 435-453.

Muñoz, Ismael, Maritza PAREdes y Rosemary ThorP

2006 «Acción Colectiva, Violencia Política y Etnicidad en el Perú». Documento de Trabajo $\mathrm{n}^{\circ}$ 1. Lima: Centro de Investigaciones Sociológicas, Económicas, Políticas y Antropológicas (CISEPA), PUCE.

NASH, June

1985 «Religión, rebelión y conciencia de clase en las comunidades mineras del estaño en Bolivia». Allpanchis 22: 115-135.

OrLove, Benjamín

1980 «Ecological Anthropology». Annual Review of Anthropology 9: 235-273.

RAPPAPORT, ROy

1975 «Naturaleza, cultura y antropología ecológica», en Hombre, cultura y sociedad, H. L. Shapiro, ed., 261-292. México: Fondo de Cultura Económica.

Rivera Cusicanqui, Silvia

1990 «Democracia liberal y democracia de ayllu: del Norte de Potosí, Bolivia», en El dificil camino hacia la democracia, pp. 9-50. La Paz: ILDIS.

Towsend, Pat

2000 Environmental Anthropology: From Pigs to Policies. Mayfield: Waveland Press. 
Valderrama Fernández, Ricardo y Carmen Escalante GutiérRez

1992 Nosotros los humanos: ñuqanchik rumakuna. Testimonio de los quechuas del siglo $X X$ [1977]. Biblioteca de la Tradición Oral Andina. Cuzco: Centro de Estudios Andinos Bartolomé de Las Casas. 